\title{
Palicourea hoffmannseggiana (Schult.) Borhidi (Rubiaceae): uma revisão de taxonomia botânica, fitoquímica, atividades biológicas e atividade antimalárica
}

\author{
Palicourea hoffmannseggiana (Schult.) Borhidi (Rubiaceae): a revision on botanical \\ taxonomy, phytochemistry, biological activities and antimalarial activity
}
Palicourea hoffmannseggiana (Schult.) Borhidi (Rubiaceae): una revisión de taxonomia botánica, fitoquímica, actividades biológicas y actividad antimalárica

Leticia Hiromi Ohashi*, Alaíde Braga de Oliveira ${ }^{1,2}$.

\begin{abstract}
RESUMO
Objetivo: Realizar uma revisão bibliográfica sobre taxonomia botânica, fitoquímica e atividades biológicas de Palicourea hoffmannseggiana (Schult.) Borhidi como base para a avaliação da atividade antimalárica da mesma. Revisão Bibliográfica: A família Rubiaceae e os gêneros Psychotria e Palicourea representam importantes fontes de produtos naturais bioativos. Botanicamente os dois gêneros são considerados morfologicamente muito próximos o que, às vezes, torna difícil a distinção entre algumas espécies. No entanto, mais recentemente, dados de filogenia molecular demonstraram que espécies tropicais do subgênero Heteropsychotria, até então pertecente ao gênero Psychotria, estão mais relacionadas a espécies tropicais do gênero Palicourea, o que resultou na reorganização taxonômica destes gêneros, com a transferência de espécies do subgênero Heteropsychotria para o gênero Palicourea. Quanto à fitoquímica, uma pesquisa bibliográfica realizada sobre Palicourea hoffmannseggiana (Schult.) Borhidi e suas sinonímias, constatou-se que a literatura registra a o isolamento e identificação de 20 metabólitos secundários, sendo 11 alcaloides que apresentaram atividades antidepressiva e anti-inflamatória e citotóxica, entre outras. Considerações finais: A revisão bibliográfica revelou citações referentes à presença de diversas substâncias potencialmente antimaláricas em Palicourea hoffmannseggiana (Schult.) Borhidi, como alcaloides, naftoquinonas e iridoides. No entanto, não se constatou nenhuma avaliação da atividade antimalárica desta espécie o que justifica o estudo proposto.
\end{abstract}

Palavras-chave: Rubiaceae, Etnofarmacologia, Plantas medicinais.

\section{ABSTRACT}

Objective: To carry out a literature revision on botanical taxonomy, phytochemistry and biological activities of Palicourea hoffmannseggiana (Schult.) Borhidi. Literature Revision: The Rubiaceae family and the genera Psychotria and Palicourea proved to be important sources of bioactive compounds. Botanically the two genera are considered morphologically very similar. However, studies on molecular phylogeny have shown that tropical species of the subgenus Heteropsychotria, until then belonging to the genus Psychotria, are more related to tropical species of the genus Palicourea, and a taxonomic reorganization of these genera was done, transferring species of the subgenus Heteropsychotria to the genus Palicourea. Regarding the phytochemistry of Palicourea hoffmannseggiana (Schult.) Borhidi and its synonyms the literature reports the isolation and identification of 20 secondary metabolites, 11 of which are alkaloids that disclosed biological activities, such as antidepressant, cytotoxic and anti-inflammatory, among others. Final considerations: The literature revision demonstrated the presence of several potentially antimalarial natural products in Palicourea hoffmannseggiana (Schult.) Borhidi, such as alkaloids, naphthoquinones and iridoides. However, no report on the antimalarial evaluation of this species and its synonims was found what justifies the proposal of investigating this effect of this plant species.

Key words: Rubiaceae, Ethnopharmacology, Medicinal plants.

1 Universidade Federal do Pará, Belém - PA. *E-mail: leticia.hiromi.ohashi@gmail.com

2 Universidade Federal de Minas Gerais, Belo Horizonte - MG.

SUBMETIDO EM: 1/2020

ACEITO EM: 2/2020

PUBLICADO EM: $\mathbf{3 / 2 0 2 0}$

REAS/EJCH | Vol.Sup.n.43 | e3027 | DOI: https://doi.org/10.25248/reas.e3027.2020 Página 1 de 11 


\section{RESUMEN}

Objetivo: Realizar una revisión sobre taxonomía botánica, fitoquímica y actividades biológicas de Palicourea hoffmannseggiana (Schult.) Borhidi. Revisión bibliográfica: La familia Rubiaceae y los géneros Psychotria y Palicourea demostraron ser fuentes importantes de compuestos potencialmente antimaláricos. Botánicamente, se consideran los dos géneros morfológicamente muy similares. Sin embargo, datos de filogenia molecular han demostrado que las especies tropicales del subgénero Heteropsychotria, hasta entonces pertenecientes al género Psychotria, están más relacionadas con las especies tropicales del género Palicourea, lo que resultó en una reorganización taxonomica de estos géneros, transfiriendo-se algunas especies del subgénero Heteropsychotria al género Palicourea. Con respecto a la fitoquímica de Palicourea hoffmannseggiana (Schult.) Borhidi y sus sinónimos, la literarura registra el aislamiento y identificación de 20 productos naturales, 11 de los cuales son alcaloides que demonstaran actividades biológicas, como antidepresivos, citotóxicos y antiinflamatorios, entre otras. Consideraciones finales: La revisón realizada demostró la presencia de diversos metabólitos secundários, potencialmente antimaláricos, en la espécie Palicourea hoffmannseggiana (Schult.) Borhidi, como alcaloides, naftoquinonas y iridoides. Sin embargo, non se encontraran registros de estudios sobre la actividad antimalárica desta espécie y sus sinonímias lo que justifica el proyeito de investigacíon de esto effecto para esta espécie vegetal.

Palabras clave: Rubiaceae, Etnofarmacología, Plantas medicinales.

\section{INTRODUÇÃO}

A malária, conhecida, também, como paludismo, impaludismo ou febre palustre, é uma doença febril aguda cujos agentes etiológicos são protozoários do gênero Plasmodium, principalmente $P$. falciparum e $P$. vivax, sendo transmitida a humanos pela fêmea infectada de mosquitos do gênero Anopheles (FRANÇA TCC, et al., 2008).

Está presente em regiões tropicais e subtropicais e representa uma das principais causas de mortes no mundo, com 228 milhões de casos e 405 mil mortes em 2018. Por se tratar de infecção parasitária humana com alto grau de morbidade e mortalidade, a malária se tornou uma prioridade para várias entidades na área internacional de saúde, como a Organização Mundial da Saúde (OMS), com o objetivo de redução dos casos de malária/paludismo que tem estado estagnada após vários anos de decréscimo a nível mundial, de acordo com o novo Relatório de 2019 da OMS sobre a malária e que define os alvos de uma estratégia técnica global para a malária até 2030, no âmbito da perspectiva de "Um mundo livre de malária" (WHO, 2019).

Com referência ao tratamento, os principais fármacos antimaláricos têm as histórias do seu desenvolvimento vinculadas ao uso tradicional de plantas medicinais, como a quinina e a artemisinina, substâncias ativas oriundas, respectivamente, de espécies pertencentes ao gênero Cinchona L. (família Rubiaceae), da América do Sul, e de Artemisia annua L. (família Asteraceae), esta última milenarmente empregada na China para tratamento de febres. A pesquisa de novos antimaláricos é um desafio para a comunidade cientifica internacional tendo em vista a resistência do $P$. falciparum, o agente etiológico da forma mais grave da malária. A investigação da atividade antimalárica de plantas constitui uma estratégia válida e promissora, principalmente no Brasil, tendo em vista a riqueza da sua biodiversidade vegetal. Diferentes abordagens, ou critérios, podem ser empregados na seleção de plantas para um estudo químico orientado para uma determinada atividade biológicalfarmacológica, entre estes a quimiotaxonomia, também conhecida como quimiosistemática ou bioquímica sistemática (MENEGUETTI DUO, et al., 2014). O vasto conhecimento sobre a fitoquímica de espécies vegetais da flora brasileira possibilita a seleção de espécies alvo como fontes de determinadas classes de produtos naturais na pesquisa de antimaláricos. Os alcaloides constituem uma vasta classe de produtos naturais apresentando uma ampla diversidade estrutural e tem mostrado atividade antimalárica (MARIATH IR, et al., 2009).

Considerando a abordagem quimiotaxonômica, com foco em alcaloides, selecionamos a família Rubiaceae e espécies do gênero Psychotria, reconhecidas produtoras de alcaloides, para um screening de atividade antimalártica. Deste gênero já foram isolados alcaloides com ampla diversidade estrutural sendo, entretanto, raras as investigações de atividade antimalárica de espécies deste gênero (CALIXTO NO, et al., 
2016) o que motivou o desenvolvimento de um projeto objetivando a avaliação da atividade antiplasmódica de espécies de Psychotria dos estados de Minas Gerais e do Pará, o qual se encontra em desenvolvimento. Um espécimen coletado no município de Marapanim, no estado do Pará, nas coordenadas S $0^{\circ} 35^{\prime} 35.23^{\prime \prime}$ W $47^{\circ} 39^{\prime} 28.92 "$ ", em área de vegetação de restinga marinha foi, inicialmente, identificado como Psychotria hoffmannseggiana (Schult.) Müll. Arg. e, posteriormente, verificou-se que este nome é, atualmente, considerado sinonímia para Palicourea hoffmannseggiana (Schult.) Borhidi (http://www.tropicos.org/Name/100408987).

A proximidade taxonômica entre os gêneros Psychotria e Palicourea (BORHIDI AL, 2011) justifica a inclusão de espécies deste último no projeto inicialmente focado em Psychotria. Portanto, o objetivo do presente artigo foi realizar uma revisão sobre taxonomia botânica, fitoquímica e atividades biológicas de Palicourea hoffmannseggiana (Schult.) Borhidi, para subsidiar o trabalho em andamento focado em atividade antiplasmódica de espécies dos gêneros Psychotria e Palicourea.

\section{REVISÃO BIBLIOGRÁFICA}

O uso de plantas com finalidade de tratar, prevenir ou curar doenças é uma das práticas medicinais mais antigas da humanidade (VEIGA JR VF, et al., 2005). Dentre as famílias com histórico de uso medicinal, inclui-se a família Rubiaceae com cerca de 640 gêneros e 10.700 espécies distribuídas nas regiões tropicais e subtropicais, podendo também ser encontradas nas regiões temperadas e frias da Europa e norte do Canadá. No Brasil, é considerada uma das principais famílias da flora brasileira, contando com cerca de 130 gêneros e 1.500 espécies (MARGALHO LF, et al., 2009).

Estudos fitoquímicos de espécies desta família revelaram uma grande variedade de classes de metabólitos secundários, como flavonoides, cumarinas, alcaloides, antraquinonas, terpenos (monoterpenos, sesquiterpenos, diterpenos e triterpenos). É considerada uma das famílias de elevada importância econômica, compreendendo plantas alimentícias, ornamentais e medicinais da flora brasileira. Entre as principais espécies com importância econômica, destaca-se o café (Coffea arabica L), bebida estimulante e tonificante que contém várias substâncias farmacologicamente ativas, como o alcaloide cafeína. Trata-se de um arbusto ou pequena árvore, originária da Etiópia, porém cultivada em diversas regiões do mundo, principalmente no Brasil (MARTINS D e NUNEZ CV, 2012; CALIXTO NO, et al., 2016).

O gênero Cinchona, pertencente à família Rubiaceae, destacando-se pela sua importância medicinal. Popularmente conhecidas como quinas, espécies deste gênero são ricas em alcaloides quinolínicos, como a quinina. Durante mais de 200 anos, a quinina foi o único fármaco antimalárico em uso clínico e o conhecimento de sua estrutura química motivou a síntese de quinolinas antimaláricas, como a cloroquina que foi introduzida na terapêutica da malária após a segunda guerra mundial (KFFURI CW, 2014).

A esta família também pertencem os gêneros Psychotria e Palicourea, ambos da tribo Psychotrieae, e que são morfologicamente muito semelhantes. O gênero Psychotria tem sido separado em dois subgêneros: Psychotria e Heteropsychotria. Entretanto, dados recentes de filogenia molecular mostraram que a tribo Psychotrieae é limitada a dois grupos monofiléticos, um deles incluindo o gênero Psychotria subgênero Psychotria e o outro incluindo, entre outros, o gênero Palicourea e algumas espécies mesoamericanas do subgênero Heteropsychotriae, cujos representantes foram transferidos para o gênero Palicourea, resultando em novas combinações e novas nomenclaturas (BREMER B e ERIKSSON T, 2009; BORHIDI AL, 2011; DELPRETE PG e KIRKBRIDE JH, 2016).

O gênero Psychotria possui importância medicinal, e é amplamente distribuído no Brasil. Psychotria viridis Ruiz \& Pav. é utilizada por povos indígenas da Amazônia como componente da bebida alucinógena conhecida como "ayahuasca", que significa "vinho da alma", consumida durante cerimônias religiosas. O chá da "ayahuasca" é uma associação das folhas de $P$. viridis e cascas de Banisteriopsis caapi, plantas ricas em $N, N$-dimetiltriptamina, um agonista não seletivo da serotonina, $5-\mathrm{HT}$, e alcalóides $\beta$-carbolinicos, respectivamente. Psychotria ipecacuanha, conhecida como ipeca e poaia, tem importância na medicina tradicional sendo usada como emética, expectorante, amebicida e antidisentérica, atividades estas 
relacionadas à presença de dois alcalóides em suas raízes: a emetina e a cefalina (CALIXTO NO, et al., 2016).

O gênero Palicourea abrange cerca de 200 espécies, sendo amplamente conhecidas na agropecuária devido à toxicidade de algumas espécies pertencentes ao gênero (TOKARNIA CH, et al., 1991). No Brasil são encontradas 74 espécies das quais 26 são consideradas endêmicas, sendo encontradas nas formas de arbustos, subarbustos e árvores. A ocorrência no Brasil é ampla, podendo ser encontradas em todas as regiões do país (FLORA DO BRASIL - http://floradobrasil.jbrj.gov.br/reflora/floradobrasil/FB14133).

Entre as espécies medicinais do gênero Palicourea citam-se $P$. coriacea (Cham.) K. Schum e $P$. rígida Kunt., que ocorrem no cerrado, no centroeste do Brasi. A primeira tem indicações para obesidade, rins e calmante. A segunda é indicada para tratamento de hipertensão, inflamação, infecção do trato urinário e do aparelho reprodutor feminino, hepatite, malária e como antiulcerogênico. Atividades diurética e antimicrobiana foram demonstradas para estas espécies, respectivamente, e nenhuma toxicidade foi observada nos animais tratados com extrato etanólico de $P$. coriacea (FREITAS PCM, et al., 2011; KATO L, et al., 2006).

\section{Nomenclatura, ocorrência e características botânicas da espécie}

Palicourea hoffmannseggiana (Schult.) Borhidi é nome aceito, conforme consta de TROPICOS (http://www.tropicos.org/Name/100408987), com várias sinonímias, entre as quais Palicourea hoffmannseggiana (Willd. ex Roem. et Schult.), Psychotria hoffmannseggiana (Willd. ex Schult.) Mull. Arg. e Psychotria barbiflora DC., cuja ocorrência é citada em países da América, como México, Equador, Colômbia, Venezuela e Brasil (DELPRETE PG e KIRKBRIDE JR. JH, 2016). A espécie é considerada nativa, porém não endêmica do Brasil, podendo ocorrer em quase todo o território brasileiro, exceto em alguns estados, como Sergipe, Piauí, entre outros, onde ainda não há relato da presença da planta na vegetação (MENDONÇA ACAM, et al., 2015). Conhecida como "erva de rato" ou "graúda", em alguns estados da região sudeste do Brasil, é uma planta considerada tóxica para animais, como bovinos, ovinos e caprinos, causando um quadro de intoxicação conhecido como "morte súbita", observada em de bovinos na África do Sul, Austrália e no Brasil. Esta espécie, juntamente com outras do mesmo gênero, como Palicourea marcgravii, $P$. longiflora e $P$. barraensis, são amplamente estudadas devido à sua toxicidade com um quadro clínico agudo que leva o animal à morte. A toxicidade de espécies de Palicourea está relacionada à presença de uma substância tóxica, o monofluoroacetato de sódio cujo mecanismo de toxicidade envolve formação do fluorocitrato, seu metabólito ativo, que bloqueia competitivamente a aconitase e o ciclo de Krebs, o que reduz a produção de ATP, interferindo na produção de energia celular e levando à falência múltipla dos órgãos (NOGUEIRA VA, et al., 2011). A carência de relatos sobre usos populares de Palicourea hoffmannseggiana (Schult.) Borhidi pode estar provavelmente relacionada à toxicidade comprovada da mesma.

Palicourea hoffmannseggiana (Schult.) Borhidi possui como sinonímia botânica os seguintes nomes Carapichea patrisii DC, Cephaelis hoffmannseggiana Schult., Cephaelis patrisii (DC.) D. Dietr. M, Cephaelis rubra Willd. ex Roem. \& Schult., Psychotria bahiensis Müll. Arg., Psychotria barbiflora DC., Psychotria flavicans Müll. Arg., Psychotria furcata DC., Psychotria heterocephala Müll. Arg., Psychotria hoffmannseggiana (Schult.) Müll. Arg., Psychotria hoffmannseggiana fo. Pubescens Steyerm., Psychotria rubra (Willd. Ex Roem. \& Schult.) Müll. Arg., Uragoga carapichea Kuntze (DELPRETE PG e KIRKBRIDE JH, 2016).

A espécie Palicourea hoffmannseggiana (Schult.) Borhidi possui como basônimo Cephaelis hoffmannseggiana Schult. (BORHIDI AL, 2011 (http://www.tropicos.org/Name/100408987) e é classificada taxonomicamente como pertencente à classe Equisetopsida C. Agardh, subclasse Magnoliidae Novák ex Takht., superordem Asteranae Takht., ordem Gentianales Juss. ex Bercht. \& J. Presl, família Rubiaceae Juss. e gênero Palicourea Aubl. A espécie é um arbusto, com altura variando entre 0,5-1m, que possui foliação microfila simples de coloração verde escura, flores bissexuadas, brancas ou arroxeadas. Pietrobom RCV, et al. (2011) fornecem descrições detalhadas das carcteríscas morfológicas desta espécie.

REAS/EJCH | Vol.Sup.n.43 | e3027 | DOI: https://doi.org/10.25248/reas.e3027.2020 Página 4 de 11 


\section{Fitoquímica e atividades biológicas}

A família Rubiaceae apresenta uma grande diversidade de metabólitos secundários, tais como, antraquinonas, terpenoides (diterpenos, triterpenos, iridoides), flavonoides, cumarinas, outros compostos fenólicos, e alcaloides de diferentes grupos estruturais, como alcaloides indólicos, sendo esta a classe mais estudada no gênero Psychotria, devido à sua associação com diversos áefeitos farmacológicos. O grande número de substâncias descritas na literatura e as atividades farmacológicas relatadas para diversas espécies desta família demonstram que Rubiaceae é uma fonte promissora de substâncias bioativas, potenciais fármacos ou protótipos de novos fármacos (CALIXTO NO, et al., 2016; MARTINS D e NUNEZ CV, 2012).

Uma análise farmacognóstica de extratos etanólicos de folhas e caules de Psychotria hoffmannseggiana (R. \& S.) Müll. Arg., coletada na Floresta Nacional do Araripe-Apodi (Ceará), por testes de coloração mostrou a presença fenóis, flavonas, flavonóis, xantonas, chalconas e auronas, nos extratos tanto dos caules quanto das folhas, taninos foram detectados apenas no extrato das folhas e leucoantocianidinas e catequinas, apenas no extrato do caule (MENDONÇA ACAM, et al., 2015)

Desta espécie já foram isolados diversos alcaloides, como o harmano (1, Figura 1), um alcaloide $\beta$ carbolínico, e o ácido estrictosidinico (2, Figura 1), um alcaloide indólico monoterpênico isolado de outras espécies dos gêneros Psychotria e Palicourea, que foi também foi isolado de Psychotria myriantha, tendo sido demonstrada sua ação no sistema 5-HT, no hipocampo de ratos, possivelmente por inibição da enzima acetilcolinesterase, agindo na transmissão dopaminérgica e serotoninérgica, o que faz sugerir que esta substância possui uma potencial atividade ansiolítica e antidepressiva (OLIVEIRA AM, et al., 2013; FARIAS FM, et al., 2012). O harmano (1) é um alcaloide conhecido por suas ações neurofarmacológicas, destacando-se os efeitos antidepressivo e neuromodulador, apresentando importante função na modulação da memória e aprendizado, além de atividade antiplasmódica, frente a cepas de Plasmodium falciparum, resistente e sensível à cloroquina (FARZIN D e MANSOURI N, 2006; ANCOLIO C, et al., 2002)

De Psychotria bahiensis, foram isolados dois alcaloides indólicos bismonoterpênicos glicosilados, 0 bahienosideo A (3, Figura 1) e o bahienosideo B (4, Figura 1) de espécimen coletado em Trindade e Tobago (PAUL JAH, et al., 2003). Estes alcaloides foram pouco estudados quanto às suas potenciais atividades biológicas e não mostraram nenhum efeito citotóxico na concentração de $10 \mu \mathrm{g} / \mathrm{ml}$ frente a células tumorais humanas de mama, fígado, cólon e pulmão (XIN WB, et al., 2011). Outras substâncias conhecidas já foram isoladas desta espécie, como estrictosamida (5, Figura 1), angustina (6, Figura 1), 5acarboxiestrictosideo (7, Figura 1), (E) e (Z)-vallesiachotamina (8a, b, Figura 1; PAUL JAH, et al., 2003).

O alcaloide estrictosamida (5, Figura 1) foi obtido a partir de extratos de raízes e galhos de Psychotria prunifolia (Kunth) Steyerm que mostraram atividade leishmanicida contra as formas promastigotas de Leishmania amazonensis (KATO L, et al., 2012). Este alcaloide é responsável pela atividade antiinflamatória de extratos brutos de Nauclea latifolia (Quadro 2; HERRMANN J, et al., 2013).

Treze alcaloides encontrados no gênero Psychotria foram avaliados quanto aos seus efeitos frente a acetilcolinesterase, butirilcolinesterase e monoaminoxidases $A$ e B (MAO A e MAO B) alvos enzimáticos relacionados a doenças neurodegenerativas. Angustina (6, Figura 1), (E) e (Z)-vallesiachotamina (8a e 8b, Figura 1) promoveram inibição das enzimas butirilcolinesterase e monoaminoxidase $A$, o que torna estes alcaloides de interesse para 0 desenvolvimento de fármacos para tratamento de neurodegeneração (PASSOS CS, et al., 2013).

Em sua dissertação, Naves RF (2014) descreve o isolamento e identificação de sinapato de metila (9, Figura 1), um éster metílico do ácido sinápico (3,5-dimetoxi-4-hidroxicinâmico); uma cumarina, a 6-hidroxi7-metoxicumarina, conhecida como isoescopoletina (10, Figura 1); cinco alcaloides, sendo três indólicos, harmano (1); $N$-metil-1,2,3,4-tetraidro- $\beta$-carbolina (11) e $N, N$-dimetiltriptamina (12), um alcalóide polindólico, a (+)-quimonantina (13), e um alcaloide indólico monoterpênico, o ácido estrictosidínico (2), além de um derivado da glicose, a $\beta$-etilglicose (14). A atividade analgésica de (+)-quimonantina (13) havia sido demonstrada anteriormente (VEROTTA L, et al., 2002). 
A N,N-dimetiltriptamina (12, Figura 1), é amplamente conhecida devido à sua ação psicoativa. Esta substância está presente em plantas utilizadas em rituais religiosos, como da União do Vegetal e Santo Daime, nos quais é consumida a bebida ayahuasca, preparada a partir das espécies Psychotria viridis e Banisteriopsis caapi (GAUJAC A, 2013).

Hayashi T, et al. (1987) isolaram de Psychotria rubra uma nova naftoquinona, a psychorubrina (15, Figura 1), que apresentou atividade citotóxica frente a células KB (carcinoma epidermóide nosofaríngeo). Isolada também de Mitracarpus frigidus (Rubiaceae), esta naftoquinona apresentou atividade antimicrobiana (Staphylococcus pyogenes e S. aureus), antitumoral contra células leucêmicas e tumor de mama, antileishmania, contra as espécies Leishmania amazonensis, L. major, L. brasiliensis e L. chagasi (LEMOS ASO, et al., 2018; FABRI RL, et al., 2012). Apresentou, também, moderada atividade antiplasmódica, porém, devido à sua alta citotoxicidade, o seu uso como alternativa terapêutica se torna inviável (ENDALE $M$, et al., 2011). Estes autores também demonstraram que a psychorubrina (15) apresentou potentes atividades antiinflamatória e antitumoral, induz a apoptose, inibe a telomerase, promove clivagem de caspase, aumenta os níveis dos marcadores autofágicos, induz a morte celular de células leucêmicas e reduz a expressão de NF-kB p65, de forma tempo e dose dependente, sendo considerada uma alternativa promissora para o tratamento de câncer (LIM CB, et al., 2012; LYSS G, et al., 1997). Desta espécie foi também isolada a helenalina (16, Figura 1), uma sesquiterpenolactona que apresentou atividade tripanocida contra epimastigotas do Trypanosoma cruzi, agente etiológico da doença de Chagas (JIMENEZORTIZ V, et al., 2005). Lu HX, et al. (2014) isolaram quatro substâncias desta espécie dois iridoides glicosilados, psyrubrina A (17, Figura 1) e 6a-hidroxigeniposídeo (18, Figura 1) e duas flavonas, 6-hidroxiluteolina-7-O-rutinosídeo (20, Figura 1) e luteolina-7-O-rutinosídeo (20, Figura 1). A luteolina-7-Orutinosídeo, isolada de outras espécies, apresentou atividade antiinflamatória, inibindo as respostas inflamatórias induzidas por lipopolissacarídeos e efeitos antimutagênicos (PARK CM e SONG YS, 2013). O iridoide 6a-hidroxigeniposídeo (18, Figura 1), um monoterpeno, demonstrou atividade imunossupressora (CHANG WL, et al., 2005). 
Quadro 1 - Substâncias isoladas de Palicourea hoffmannseggiana (Schult.) Borhidi e suas sinonímias

\begin{tabular}{|c|c|c|c|}
\hline Classe metabólica & Espécie & Substância & Referência \\
\hline \multirow{10}{*}{ Alcaloides } & Psychotria bahiensis DC. & 5a-carboxiestrictosidina (7) & PAUL JAH, et al., 2003 \\
\hline & Psychotria hoffmannseggiana Roem. \& Schult & (+)-quimonantina (13) & NAVES RF, 2014 \\
\hline & Psychotria bahiensis DC. & $(E) /(Z)-$ vallesiachotamina (8) & PAUL JAH, et al., 2003; \\
\hline & $\begin{array}{l}\text { Psychotria hoffmannseggiana Roem. \& Schult; } \\
\text { Psychotria barbiflora DC. }\end{array}$ & ácido estrictosidinico (2) & $\begin{array}{l}\text { OLIVEIRA AM, et al., 2013; } \\
\text { NAVES RF, 2014; }\end{array}$ \\
\hline & Psychotria bahiensis DC. & angustina (6) & PAUL JAH, et al., 2003 \\
\hline & Psychotria bahiensis DC. & bahienosida A (3) e B (4) & PAUL JAH, et al., 2003 \\
\hline & Psychotria bahiensis DC. & estrictosamida (5) & PAUL JAH, et al., 2003 \\
\hline & $\begin{array}{l}\text { Psychotria hoffmannseggiana Roem. \& Schult; } \\
\text { Psychotria barbiflora DC. }\end{array}$ & harmano (1) & $\begin{array}{l}\text { OLIVEIRA AM, et al., 2013; } \\
\text { NAVES RF, } 2014\end{array}$ \\
\hline & Psychotria hoffmannseggiana Roem. \& Schult & $N$-metil-1,2,3,4-tetraidro- $\beta$-carbolina (11) & NAVES RF, 2014 \\
\hline & Psychotria hoffmannseggiana Roem. \& Schult & $N, N$-dimetiltriptamina (12) & NAVES RF, 2014 \\
\hline Cumarina & Psychotria hoffmannseggiana Roem. \& Schult & isoescopoletina (10) & NAVES RF, 2014 \\
\hline \multirow{2}{*}{ Flavonoides } & Psychotria rubra* & 6-hidroxi-luteolina-7-O- rutinosídeo (19) & LU HX, et al., 2014; \\
\hline & Psychotria rubra* & luteolina-7-O-rutinosídeo (20) & LU HX, et al., 2014 \\
\hline \multirow{2}{*}{ Terpenos } & Psychotria rubra* $^{*}$ & 6a-hidroxigeniposídeo (18) & LU HX, et al., 2014 \\
\hline & Psychotria rubra* $^{*}$ & psyrubrina A (17) & LU HX, et al., 2014 \\
\hline Quinonas & Psychotria rubra* $^{*}$ & psychorubrina (15) & HAYASHI T, et al., 1987 \\
\hline Terpenos & Psychotria rubra* & helenalina (16) & HAYASHI T, et al., 1987 \\
\hline \multirow{2}{*}{ Outras classes } & Psychotria hoffmannseggiana Roem. \& Schult & $\beta$-etilglicose (14) & NAVES RF, 2014 \\
\hline & Psychotria hoffmannseggiana Roem. \& Schult & sinapato de metila (9) & NAVES RF, 2014 \\
\hline
\end{tabular}

Legenda: *Classificadores botânicos não informados na fonte citada.

Fonte: Ohashi LH e Oliveira AB, 2019.

REAS/EJCH | Vol.Sup.n.43 | e3027 | DOI: https://doi.org/10.25248/reas.e3027.2020 Página 7 de 11 
Quadro 2 - Atividades biológicas das substâncias isoladas de Palicourea hoffmannseggiana (Schult.) Borhidi e suas sinonímias.

\begin{tabular}{|c|c|c|}
\hline Substância & Atividade biológica & Referência \\
\hline 5a-carboxiestrictosidina (7) & *** & PAUL JAH, et al., 2003 \\
\hline (+)-quimonantina (13) & Analgésico & NAVES RF, 2014 \\
\hline$(E) /(Z)$-vallesiachotamina (8) & $\begin{array}{l}\text { Inibidor das enzimas butirilcolinesterase } \mathrm{e} \\
\text { monoaminoxidase A (MAO A) }\end{array}$ & PAUL JAH, et al., 2003; PASSOS CM, et al., 2013 \\
\hline ácido estrictosidinico (2) & $\begin{array}{lccc}\begin{array}{l}\text { Ansiolítico } \\
\text { acetilcolinesterase }\end{array} & \text { antidepressivo; } & \text { Inibidor } \mathrm{da} \\
\end{array}$ & $\begin{array}{l}\text { OLIVEIRA AM, et al., 2013; NAVES RF, 2014; FARIAS FM, } \\
\text { et al., } 2012\end{array}$ \\
\hline angustina (6) & $\begin{array}{lll}\text { Inibidor das enzimas butirilcolinesterase } & e \\
\text { monoaminoxidase A (MAO A) } & \\
\end{array}$ & PAUL JAH, et al., 2003; PASSOS CM, et al. 2013 \\
\hline harmano (1) & Antidepressivo; neuromodulado; antiplasmódico & $\begin{array}{l}\text { OLIVEIRA AM, et al., 2013; NAVES RF, 2014; FARZIN D e } \\
\text { MANSOURI N, 2006; ANCOLIO C, et al., 2002. }\end{array}$ \\
\hline $\begin{array}{l}N \text {-metil-1,2,3,4-tetraidro- } \beta \text {-carbolina } \\
\text { (11) }\end{array}$ & *** & NAVES RF, 2014 \\
\hline luteolina-7-O-rutinosídeo (20) & Antiinflamatório; antimutagênico & LU HX, et al., 2014; PARK CM e SONG YS, 2013 \\
\hline 6a-hidroxigeniposídeo (18) & Imunossupressor & LU HX, et al., 2014; CHANG WL, et al., 2005 \\
\hline psyrubrina A (17) & *** & LU HX, et al., 2014; \\
\hline psychorubrina (15) & $\begin{array}{l}\text { Citotóxico (Células KB); antibacteriano e antibiofilme; } \\
\text { antiplasmódico; antileishmania }\end{array}$ & $\begin{array}{l}\text { HAYASHI T, et al., 1987; ENDALE M, et al., 2011; LEMOS } \\
\text { ASO, et al. 2018, FABRI RL, et al., } 2012\end{array}$ \\
\hline helenalina (16) & $\begin{array}{l}\text { Anticancerígeno; } \\
\text { antileucêmico }\end{array}$ tripanocida; $\quad$ antitumoral; & $\begin{array}{l}\text { HAYASHI T, et al., 1987; LIM CB, et al., 2012; JIMENEZ- } \\
\text { ORTIZ V, et al., 2005; LYSS G, et al., } 1997\end{array}$ \\
\hline$\beta$-etilglicose (14) & *** & NAVES RF, 2014 \\
\hline sinapato de metila (9) & *** & NAVES RF, 2014 \\
\hline
\end{tabular}

Legenda: ${ }^{* * *}$ Não foram encontrados estudos de atividade biológica na literatura. Fonte: Ohashi LH e Oliveira, AB, 2019. 
Figura 1 - Estruturas químicas das substâncias isoladas de Palicourea hoffmannseggiana (Schult.) Borhidi e suas sinonímias (Quadros 1 e 2)
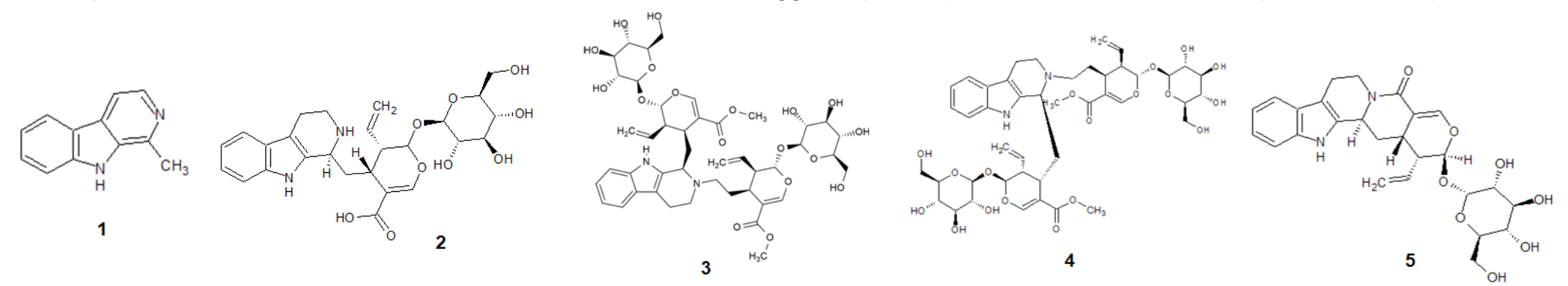

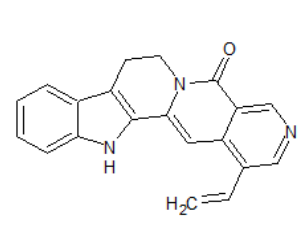

6
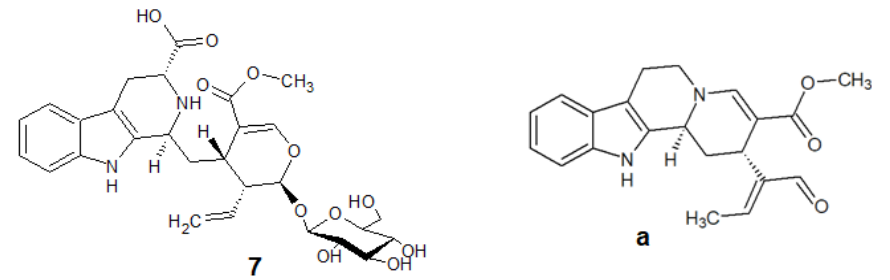

a

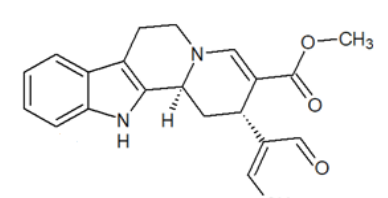

b<smiles>COC(=O)/C=C/c1cc(OC)c(O)c(OC)c1</smiles>

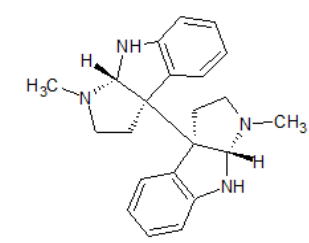

13

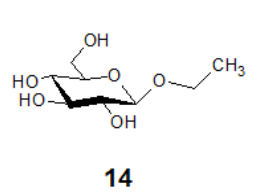

14

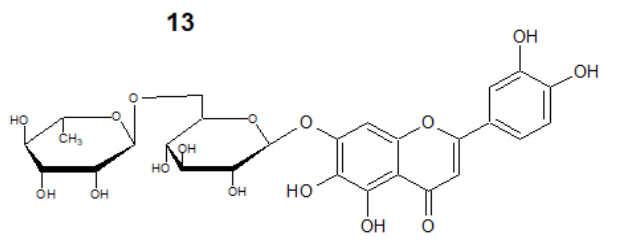

19

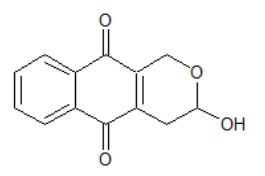

15

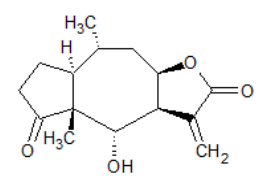

16
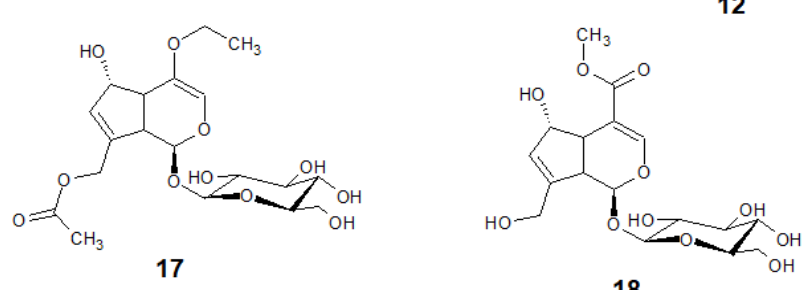

18

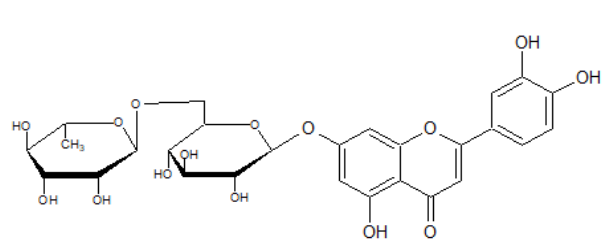

20

Legenda: 1: harmano; 2: ácido estrictosidínico; 3: bahienosídeo A; 4: bahienosídeo B; 5: estrictosamida; 6: angustina; 7: 5a-carboxiestrictosidina; 8a: (E)vallesiachotamina; 8b: (Z)-vallesiachotamina; 9: sinapato de metila; 10: isoescopoletina; 11: $N$-metil-1,2,3,4-tetraidro- $\beta$-carbolina; 12: $N, N$-dimetiltriptamina; 13: (+)quimonantina; 14: $\beta$-etilglicose; 15: psychorubrina; 16: helenalina; 17: psyrubrina A; 18: 6a -hidroxigeniposídeo; 19: 6-hidroxi-luteolin-7-O- rutinosídeo; 20: luteolina7-O-rutinosídeo. Fonte: Ohashi LH e Oliveira AB, 2019.

REAS/EJCH | Vol.Sup.n.43 | e3027 | DOI: https://doi.org/10.25248/reas.e3027.2020 Página 9 de 11 


\section{CONSIDERAÇÕES FINAIS}

Esta revisão bibliográfica revelou citações referentes à presença de diversas substâncias potencialmente antimaláricas em Palicourea hoffmannseggiana (Schult.) Borhidi, principalmente alcaloides dos grupos dos indolomonoterpênicos ainda não avaliados quanto à sua atividade antimalárica, além de um alcaloide do tipo $\beta$-carbolínico, o harmano (1), de atividade comprovada, o que justifica a investigação desta espécie como fonte de potenciais alcaloides antimaláricos. A conhecida toxicidade da espécie, relacionada à presença de monofluoroacetato, em espécimens previamente avaliados, não constitui impedimento para a sua investigação no âmbito do objetivo proposto uma vez que a substância tóxica identificada é um sal do ácido monofluoroacético, solúvel em água, podendo ser facilmente separado dos alcaloides descritos para esta espécie. Além disso, não foram encontrados registros de avaliação da atividade antimalárica de Palicourea hoffmannseggiana (Schult.) Borhidi, das suas sinonímias, dos alcaloides presentes nestas e em outras espécies taxonomicamente relacionadas, o que justifica o objetivo proposto para um projeto em andamento.

\section{AGRADECIMENTOS E FINANCIAMENTO}

Os autores agradecem à Coordenação de Aperfeiçoamento de Pessoal de Nível Superior - Brasil (CAPES) e ao Conselho Nacional de Desenvolvimento Científico e Tecnológico - Brasil (CNPq) pelas bolsas concedidas a LHO (Mestrado, PPGCF UFPA), ABO (Pesquisador Sênior - PQ-Sr, CNPq; PVNS CAPES).

\section{REFERÊNCIAS}

1. ANCOLIO C, et al. Antimalarial Activity of Extracts and Alkaloids Isolated from Six Plants Used in Traditional Medicine in Mali and São Tomé. Phytotherapy Research, 2002; 16: 646-649.

2. BORHIDI, A. Transfer of the Mexican species of Psychotria subgen. Heteropsychotria to Palicourea based on morphological and molecular evidences. Acta Botanica Hungarica, 2011; 53(3-4): 241-250.

3. BREMER B e ERIKSSON T. Time Tree of Rubiaceae: Phylogeny and Dating the Family, Subfamilies, and Tribes. International Journal of Plant Sciences, 2009; 170(6): 766-793.

4. CALIXTO NO, et al. The Genus Psychotria: Phytochemistry, Chemotaxonomy, Ethnopharmacology and Biological Properties. Journal of the Brazilian Chemical Society, 2016; 27(8): 1355-1378.

5. CHANG WL, et al. Immunosuppressive Iridoids from the Fruits of Gardenia jasminoides. Journal of Natural Products, 2005; 68: 1683-1685

6. DELPRETE PG e KIRKBRIDE JR. JH. New combinations and new names in Palicourea (Rubiaceae) for species of Psychotria subgenus Heteropsychotria occurring in the Guianas. Journal of the Botanical Research Institute of Texas, 2016; 10(2): 409-442.

7. ENDALE M, et al. Antiplasmodial Quinones from Pentas longiflora and Pentas lanceolata, Planta Médica, 2012; 78 : $31-35$

8. FABRI RL, et al. Antitumor, Antibiotic and Antileishmanial Properties of the Pyranonaphthoquinone Psychorubrin from Mitracarpus frigidus, Anais da Academia Brasileira de Ciências, 2012; 84(4): 1081-1089.

9. FARIAS FM, et al. Strictosidinic acid, isolated from Psychotria myriantha Mull. Arg. (Rubiaceae), decreases serotonin levels in rat hippocampus. Fitoterapia, 2012; 83: 1138-1143.

10. FARZIN D, MANSOURI N. Antidepressant-like effect of harmane and other $\beta$-carbolines in the mouse forced swim test. European Neuropsychopharmacology, 2006; 16(5): 324-328.

11. FERREIRA Jr. M, VIEIRA AOS. Espécies arbóreo-arbustivas da família Rubiaceae Juss. na bacia do rio Tibagi, PR, Brasil. Hoehnea, 2015; 42(2): 289-336.

12. FLORA DO BRASIL. Rubiaceae in Flora do Brasil 2020. Jardim Botânico do Rio de Janeiro. Disponível em: <http://floradobrasil.jbrj.gov.br/reflora/floradobrasil/FB14133>. Acesso em: 16 Jan. 2020.

13. FRANÇA TCC, et al. Malária: Aspectos históricos e quimioterapia. Quimica Nova, 2008; 31(5): 1271-1278

14. FREITAS PCM, et al. Diuretic activity and acute oral toxicity of Palicourea coriacea (Cham.) K Schum. Journal of Ethnopharmacology, 2011; 134(2): 501-503.

15. GAUJAC A. Estudos sobre o psicoativo N,N-Dimetiltriptamina (DMT) em Mimosa tenuiflora (Willd.) Poirt e em bebidas consumidas em contexto religioso. Tese (Doutorado em química) - Programa de Pós-Graduação em Química. Universidade Federal da Bahia, Salvador, 2013; 195 p. 
16. HAYASHI T, et al. Antitumor Agents. Psychorubrin, a New Cytotoxic Naphthoquinone from Psychotria rubra and Its Structure-Activity Relationships. Journal of Medicinal Chemistry, 1987; 30(11): 2005-2008.

17. JIMENEZ-ORTIZ V, et al. The trypanocidal effect of sesquiterpene lactones helenalin and mexicanin on cultured epimastigotes. Journal of Parasitology, 2005; 91(1): 170-174

18. KATO L, et al. Antiprotozoal alkaloids from Psychotria prunifolia (Kunth) Steyerm. Journal of the Brazilian Chemical Society, 2012; 23(2): 355-360.

19. KFFURI CW. Etnobotânica de plantas antimaláricas em comunidades indígenas da região do alto Rio Negro Amazonas - Brasil. Tese (doutorado) - Faculdade de Ciências Agronômicas. Universidade Estadual Paulista, São Paulo, 2014; 2013 p.

20. LEMOS ASO, et al., Antibacterial and Antibiofilm Activities of Psychorubrin, a Pyranonaphthoquinone Isolated From Mitracarpus frigidus (Rubiaceae). Frontiers in Microbiology, 2018; 9: 1-11.

21. LIM CB, et al. NF-KB p65 repression by the sesquiterpene lactone, Helenalin, contributes to the induction of autophagy cell death. Complementary and Alternative Medicine, 2012; 12 : 93.

22. LU HX, et al. A new iridoid glycoside from the root of Psychotria rubra. Biochemical Systematics and Ecology, 2014; 57: 133-136

23. LYSS G, et al. Helenalin, an Anti-Inflammatory Sesquiterpene Lactone from Arnica, Selectively Inhibits Transcription Factor NF-KB. Biological Chemistry, 1997; 378: 951 -961.

24. MARGALHO LF, et al. Rubiaceae Juss. da restinga da APA de Algodoal, Maiandeua, Maracanã, Pará, Brasil. Boletim do Museu Paraense Emílio Goeldi. Ciências Naturais, 2009: 4(3): 303-339.

25. MARIATH IR, et al. Plants of the American continent with antimalarial activity. Revista Brasileira de Farmacognosia, 2009; 19(1A): 158-192

26. MARTINS D, NUNEZ CV. Secondary Metabolites from Rubiaceae Species. Molecules, 2015; 20:13422-13495.

27. MENDONÇA ACAM, et al. Prospecção fitoquímica de Psychotria colorata (willd. Ex. R. \& s.) Müll. Arg. e P. hoffmannseggiana (r. \& s.) Müll. Arg. Caderno de Cultura e Ciência, 2015; 13(2): 7-16

28. MENEGUETTI DUO, et al. Antimalarial ethnopharmacology in the Brazilian Amazon. Revista de Ciências Farmacêuticas Básica e Aplicada, 2014; 35(4): 577-587.

29. NAVES RF. Estudo fitoquimico das folhas de Psychotria hoffmannseggiana Roem. \& Schult (Rubiaceae). Dissertação (Mestrado). Universidade Federal de Goias. Instituto de Quimica, Goiânia, 2014; 212 f.

30. NOGUEIRA VA, et al. Intoxicação por monofluoroacetato em animais. Pesquisa Veterinária Brasileira, $2011 ; 31$ (10): 823-838.

31. OLIVEIRA AM, et al. $\beta$-Carboline alkaloids from Psychotria barbiflora DC. (Rubiaceae). Biochemical Systematics and Ecology, 2013; 50: 339-341.

32. PARK CM, SONG YS. Luteolin and luteolin-7-O-glucoside inhibit lipopolysaccharide-induced inflammatory responses through modulation of NF-KB/AP-1/PI3K-Akt signaling cascades in RAW 264.7 cells. Nutrition Research and Practice, 2013; 7(6): 423-429.

33. PASSOS CS, et al. Indole alkaloids of Psychotria as multifunctional cholinesterases and monoamine oxidases inhibitors. Phytochemistry, 2013; 86: 8-20

34. PAUL JAH, et al. Novel Bis(monoterpenoid) Indole Alkaloids from Psychotria bahiensis. Journal of Natural Products, 2003; 66: 752-754.

35. PIETROBOM RCV, et al. Morfoanatomia foliar de Psychotria hoffmannseggiana (Willd. ex Roem. \& Schult.) Müll. Arg. e Psychotria trichophora Müll. Arg. (Rubiaceae). Naturalia, 2011; 34: 21-42.

36. TOKARNIA CH, et al. Intoxicação experimental por Palicourea marcgravii (Rubiaceae) em caprinos. Pesquisa Veterinária Brasileira, 1991; 3: 209-215.

37. Tropicos.org. Missouri Botanical Garden. Disponível em: http://www.tropicos.org/Name/100408987 . Acessado em 15 de jan. de 2020.

38. VEIGA JUNIOR VF, et al. Plantas medicinais: cura segura? Química Nova, 2005; 28(3): 519-528.

39. World Health Organization. World malaria report 2019. WHO, 2019.

40. XIN WB, et al. Bis(monoterpenoid) indole alkaloid glucosides from Uncaria hirsuta. Phytochemistry Letters, 2011; 4: 380-382. 